\title{
Multifunctional Micellar Nanomedicine for Cancer Therapy
}

\author{
Elvin Blanco ${ }^{1}$, Chase W. Kessinger ${ }^{1}$, Baran D. Sumer ${ }^{2}$, and Jinming Gao ${ }^{1,}$, \\ ${ }^{1}$ Department of Pharmacology, Simmons Comprehensive Cancer Center, University of Texas \\ Southwestern Medical Center at Dallas, Dallas, TX 75390 \\ 2 Department of Otolaryngology, Southwestern Medical School, University of Texas Southwestern \\ Medical Center at Dallas, Dallas, TX 75235
}

\begin{abstract}
Polymeric micelles are supramolecular, core-shell nanoparticles that offer considerable advantages for cancer diagnosis and therapy. Their relatively small size $(10-100 \mathrm{~nm})$, ability to solubilize hydrophobic drugs as well as imaging agents, and improved pharmacokinetics provide a useful bioengineering platform for cancer applications. Several polymeric micelle formulations are currently undergoing phase I/II clinical trials, which have shown improved antitumor efficacy and reduced systemic toxicity. This minireview will focus on recent advancements in the multifunctional design of micellar nanomedicine with tumor targeting, stimulated drug release, and cancer imaging capabilities. Such functionalization strategies result in enhanced micellar accumulation at tumor sites, higher drug bioavailability, as well as improved tumor diagnosis and visualization of therapy. Ultimately, integrated nanotherapeutic systems (e.g., theranostic nanomedicine) may prove essential to address the challenges of tumor heterogeneity and adaptive resistance to achieve efficacious treatment of cancer.
\end{abstract}

\section{Keywords}

Polymeric micelles; cancer nanomedicine; drug delivery systems; tumor imaging; cancer targeting; controlled release

\section{Introduction}

Cancer remains as one of the leading causes of mortality worldwide, affecting over 10 million new patients every year. Currently, the treatment options include surgical resection, radiation, and chemotherapy. However, although over 90 chemotherapeutic drugs have been approved by the FDA for clinical use, their efficacy has been severely hindered by dose-limiting toxicity and patient morbidity. Recently, nanoscale $(10-200 \mathrm{~nm})$ therapeutic systems have emerged as novel therapeutic modalities for cancer treatment (1-3). These systems include polymeric micelles, polymer-drug conjugates, dendrimers, liposomes, and inorganic particulates. Compared to conventional small molecule-based therapy, nanotherapeutic systems have several potential advantages for cancer therapy, including higher payload capacity, prolonged blood circulation times, reduced toxicity to healthy tissues, and improved anti-tumor efficacy. In this article, we will review key advances of one of these emerging nanotherapeutic systems, polymeric micelles (4-7), and discuss their potential for cancer therapy.

\footnotetext{
* To whom correspondence should be addressed: Phone: 1-214-645-6370 Fax: 1-214-645-6347 jinming.gao@utsouthwestern.edu .
} 


\section{Polymeric Micelles: Properties and Advantages for Cancer Treatment}

The use of polymeric micelles for cancer treatment was first reported in the early 1980s by Ringsdorf and coworkers (8). These spherical particles are nanosized (typically in the range of 10-100 nm) supramolecular constructs (Fig. 1) formed from the self-assembly of biocompatible amphiphilic block copolymers in aqueous environments (9). In water, the hydrophobic portion of the block copolymer self-associates into a semi-solid core, with the hydrophilic segment of the copolymer forming a coronal layer. The resulting core-shell architecture is important for drug delivery purposes, because the hydrophobic core can act as a reservoir for water insoluable drugs, while the outer shell protects the micelle from rapid clearance (10). Although several functional aspects of the constituent blocks have been explored (e.g. temperature or $\mathrm{pH}$ sensitive blocks), the most important criteria are biocompatibility and/or biodegradability. Currently, the most commonly used corona-forming polymer is polyethylene glycol (PEG), with a molecular weight range from 2 to $15 \mathrm{kD}$. Coreforming blocks typically consist of poly(propylene oxide) (PPO), poly(D,L-lactic acid) (PDLLA), poly ( $\varepsilon$-caprolactone) (PCL), and poly(L-aspartic acid) to name a few (6).

Given their lipophilic nature, most anticancer drugs are inherently water insoluble. As an example, paclitaxel, a highly effective anticancer agent that inhibits microtubule growth by binding to the $\beta$ subunit of tubulin, has a water solubility of $0.0015 \mathrm{mg} / \mathrm{mL}$. While this degree of hydrophobicity is favorable for drug permeation through cell membranes, intravenous (i.v.) administration would result in rapid drug aggregation and formation of capillary embolisms (11). By encapsulation of the drug within the hydrophobic core of the micelle, the apparent solubility of the drug can be significantly increased. For example, micelle encapsulation of paclitaxel increased the solubility over three orders of magnitude from 0.0015 to $2 \mathrm{mg} / \mathrm{mL}$ (12). Hence, polymer micelles allow for the in vivo use of previously existing drugs otherwise deemed too hydrophobic or toxic, without having to manipulate the chemical structure of the agent. Additionally, encapsulating the drug within the polymer core affords drug stability by hindering enzymatic degradation and inactivation.

The hydrophilic micellar corona also plays an important role in in vivo applications by reducing particle recognition by opsonin proteins (13). In the absence of this brush-like coating, the micelle would undergo rapid phagocytic clearance by the reticuloendothelial system (RES) (14). Additionally, the critical micelle concentration (CMC, the concentration threshold of polymers at which micelles are formed) is very low for polymeric micelles, typically on the order of $10^{-6}-10^{-7} \mathrm{M}$, resulting in stable constructs that are not easily dissociable in vivo (15). These characteristics together contribute to longer blood circulation times, and this longevity results in an increase in the bioavailability of the drug. The long circulation times and small size of polymer micelles also aid in the preferential accumulation of micelles in tumor tissue through the enhanced permeability and retention (EPR) effect, which allows for passive targeting due to fenestrations between endothelial cells in angiogenic tumor vessels $(16,17)$.

\section{Comparison of Polymeric Micelles to Other Nanotherapeutic Systems}

Polymer-drug conjugates (18), dendrimers (19) and liposomes (20) represent other major polymer-based nanotherapeutic systems, each with different chemical structures and biological properties. Among these systems, polymer-drug conjugates and liposomes have a longer history of development and to this date have found the most success in the clinics. For example, SMANCS, a conjugate of neocarzinostatin (NCS) and poly(styrene-co-maleic acid) (SMA), was developed by Maeda and coworkers in the 1980s, and has been clinically approved for liver cancer treatment (21). The blood half-life of SMANCS is 10 times higher than that of NCS, which leads to enhanced tumor targeting via the EPR effect. Most importantly, the 
improved stability and tumor selectivity resulted in increased antitumor efficacy during hepatocellular carcinoma treatment (22). Currently, other types of polymer-drug conjugates are also gaining prominence, with dextran-doxorubicin, PEG-camptothecin, and polyglutamate-paclitaxel conjugates in phase I, II, and III clinical trials (1). With regards to liposomal delivery systems, a doxorubicin-containing, PEGylated formulation, Doxil®, has been clinically approved to treat Kaposi's sarcoma and several types of solid tumors (23). While dendrimers have yet to find their way into clinical use, preliminary research with methotrexate-containing polyamidoamine dendrimers has shown growth reduction of subcutaneous tumors in mice (24). Several micellar systems are currently in Phase I/II clinical trials for the delivery of doxorubicin (DOX) and paclitaxel. Among these, Kataoka and coworkers prepared DOX micelles from a poly(ethylene glycol)-poly(L-aspartic acid) block copolymer, resulting in significantly improved preclinical antitumor efficacy (25). The micelle formulation, currently in clinical trials under the name NK911, nearly tripled the half-life of free drug (from 48 minutes to 2.3-2.8 hours) and reduced the clearance of the drug (26).

Table 1 shows the basic structures and properties of these nanoplatforms, serving to highlight differences that exist among them. Both polymer-drug conjugates and dendrimer systems necessitate the covalent conjugation of drug molecules to the carriers (27). This in turn would require the presence of functionalizable chemical groups on the drug molecules, limiting the generality of this approach. In light of the high chemical stability of covalent bonds, specific chemical strategies (e.g. enzymatic degradation, acid-catalyzed hydrolysis) are necessary to release the drug molecules at tumor sites (28). Moreover, due to the small size of these systems (typically $<10 \mathrm{~nm}$ ), they can easily cross basement membranes in the glomeruli of kidneys and be quickly cleared, leading to much shortened blood half-lives $(3,29)$.

Liposomes are vesicular nanostructures self-assembled from the phospholipid and cholesterol molecules that typically form cell membranes (20). As a result of their inner hydrophilic compartment, liposomes are more suitable for the delivery of water-soluble agents such as therapeutic proteins or DNAs. Poorly soluble drugs can be entrapped within the hydrophobic bilayer membrane, but the loading capacity is limited due to membrane destabilization effects (30). Stealth liposomes, where hydrophilic polymers such as PEG have been conjugated on the liposomal surface, have considerably prolonged blood circulation times, and effective passive targeting to solid tumors through the EPR effect has been noted in numerous studies $(31,32)$. However, due to intrinsic structural constraints, most liposomal particles are over 90 $\mathrm{nm}$ in diameter, which may considerably limit their transport in tumor tissues. For example, Yuan and coworkers have shown limited liposomal penetration to only $30 \mu \mathrm{m}$ (a few cell layers) following particle extravasation (33). Later studies have shown that fibril collagen is the main structural barrier for interstitial transport (34). Moreover, drug release from conventional liposomal formulations is quite limited once these particles reach the tumor target. To overcome this problem, thermo- and $\mathrm{pH}$-sensitive liposomes have been explored to provide responsive release of drugs in hopes of improving bioavailability $(35,36)$.

Polymeric micelles provide a unique and complementary nanoplatform to the above nanosystems for drug delivery applications. The hydrophobic cores of micelles provide a natural carrier environment that allows easy encapsulation of poorly soluble anticancer drugs. The non-covalent encapsulation strategy makes it feasible to entrap drugs without the requirement of reactive chemical groups. Meanwhile, the unique chemistry of the polymer constituents does allow for the chemical conjugation of anticancer drugs, such as doxorubicin $(37,38)$, to these chains, effectively enhancing drug loading and hindering premature drug release upon administration. Additionally, the size of polymeric micelles, 10-100 nm, can be easily controlled by varying the hydrophobic block of the amphiphilic copolymer (39). This size range also permits for evasion of renal filtration while allowing for increased tumor penetration compared to liposomes (7). 
Despite many advantages of polymer micelles for in vivo applications, several challenges exist and represent an active field of research. For example, the small micellar size of 10-100 nm limits the amount of drug that can be incorporated within the core, with higher drug loading coming at the cost of increased micelle size and aggregation $(40,41)$. This small size and limited drug loading in turn results in faster release from the micelles (42), which may cause premature release prior to the micelle reaching its intended site of action. Therefore, chemical conjugation strategies, as mentioned above, as well as increasing the compatibility of the micelle core with the intended drug (43), are being investigated to address these concerns. Additionally, several questions have been raised regarding the long-term stability of polymer micelles (9). In response to these issues, several groups are examining core-crosslinked micelles in order to enhance in vivo stability $(44,45)$. Finally, questions regarding the antitumor efficacy of micelles in the clinical setting have been raised. In this regard, many labs have explored methods to ensure accumulation of the micelles at the tumor site and release its contents in a controlled, predetermined fashion. In the sections below, we will discuss strategies employed to achieve micelle multifunctionalization, namely active targeting, stimulated drug release, and imaging sensitivity for cancer applications (Fig. 2).

\section{Active Targeting of Micelles}

Active targeting strategies, which involve the functionalization of the micelle surface with a ligand that recognizes tumor-specific receptors, are an intense area of study with several potential advantages. These include increased accumulation at tumor sites as well as increased uptake into cancer cells via receptor-mediated endocytosis (46). Commonly used ligands are grouped into the following classes: small organic molecules, peptides, carbohydrates, monoclonal antibodies, and DNA/RNA aptamers.

An example of a small organic molecule for cancer targeting applications is folic acid, whose receptor is over-expressed (100-300 times) in a variety of tumors (47). Park and coworkers functionalized DOX-containing PEG-PLGA micelles with folic acid and were able to show significantly increased uptake and cytotoxicity in KB cells (38). In vivo studies showed that folate-labeled micelles led to a 2 -fold decrease in tumor growth rate compared to non-targeted micelles. Peptides are also actively explored as ligands for tumor-targeted drug delivery. Recent work by our laboratory has investigated the use of cyclic(Arg-Gly-Asp-D-Phe-Lys) (cRGDfK) peptide, which targets the $\alpha_{v} \beta_{3}$ integrin overexpressed on the surface of angiogenic tumor vessels (48).

Carbohydrate molecules, such as galactose and lactose, have also been used to functionalize micelles. These ligands have high affinity for the asialoglycoprotein receptor (ASGPR) overexpressed in hepatocellular carcinoma (49). A galactose-labeled poly(ethylene glycol)co-poly ( $\gamma$-benzyl L-glutamate) block copolymer was used by Cho and coworkers to produce micelles encapsulating paclitaxel, and exhibited a 30\% increased uptake in ASGPR cells (50). Monoclonal antibodies represent another wide class of active targeting ligands. Recently, Torchilin and coworkers reported diacyllipid-PEG (PE-PEG) micelles conjugated with an anticancer monoclonal antibody (mAb 2C5) or an anti-myosin mAb 2G4 antibody to target lung cancer cells (11). Micelles encoded with 2C5 were able to increase paclitaxel accumulation (four-fold after $2 \mathrm{~h}$ ) and cytotoxicity in lung tumors over control micelles. Finally, tumorspecific aptamers, DNA or RNA oligonucleotides identified by library screening, are also gaining potential as targeting ligands. Docetaxel-loaded PEG-PLGA micelles were recently conjugated with an RNA aptamer specific for the prostate specific membrane antigen (PSMA) to treat prostate tumors (51). In vivo studies in $\mathrm{LNCaP}$ xenografts showed overall increased anti-tumor efficacy and lesser systemic toxicity than non-targeted micelles, and more importantly, total tumor regression in five of seven mice was reported. 


\section{Stimulus Responsive Drug Release}

Upon entering the tumor site, it is desirable that the therapeutic agent be released from the micelles in a controlled fashion in order to reach cytotoxic levels. To achieve this, several strategies have been explored that include $\mathrm{pH}$-, temperature-, and ultrasound-stimulated release.

It is now well known that tumor tissues tend to have lower $\mathrm{pH}$ values (as low as 5.7) than normal tissue environments ( $\mathrm{pH} 7.4$ ), due to the glycolysis metabolism of cancer cells. Additionally, the process of endocytosis, or the sequestration of the nanocarriers into vesicles (e.g. late endosomes, and heavily degradative lysosomes) is one associated with low $\mathrm{pH}$ values of $~ 5.0-5.5$. Hence, changes in $\mathrm{pH}$ values encountered by micelles upon intravenous injection provide a possible venue through which to achieve stimulated release of drugs. Two different strategies have been reported to induce $\mathrm{pH}$-sensitive release of drugs from micelles. These include the use of acid-labile bonds and non-covalent strategies involving selective protonation of $\mathrm{pH}$-sensitive components inside the micelle. In the first strategy, Kataoka and coworkers were able to formulate micelles where doxorubicin was conjugated to the PEG-pAsp copolymer via a hydrazone linkage (37). The resulting micelles had high loading of DOX $(42.5 \%)$ and $\mathrm{pH}$ sensitive release; $3 \%$ of the drug was released after $48 \mathrm{~h}$ in $\mathrm{pH} 7.4$ and $25 \%$ release of drug was achieved at the same time at $\mathrm{pH}$ 5.5. In vivo studies showed increased tumor accumulation, greater tolerance for the drug, and tumor regression in $50 \%$ of mice. Noncovalent strategies for $\mathrm{pH}$-sensitive release were explored by several groups (52-54). For example, Tang et al. devised a triblock polymer of PEG, poly(2-(dimethylamino) ethyl methacrylate) (DMA), and poly(2-diethylamino)ethyl acrylate (DEA) resulting in a system that dissolves completely in acidic solution but forms micelles at high $\mathrm{pH}$ (pH 8.0) (54). Acid sensitive release of dipyridamole was observed with a $50 \%$ increase of drug release at $\mathrm{pH} 3.0$ over that at $\mathrm{pH} 7.4$.

Technologies that permit for site-specific elevation of temperature have led to the development of heat-sensitive polymer micelles. The polymer of choice is poly(N-isopropylacrylamide) or pNIPAM, which has a lower critical solution temperature (LCST) of $32{ }^{\circ} \mathrm{C}$ (55). Okano and coworkers reported micelles where poly(butyl methacrylate) (PBMA) was used to form the hydrophobic core while pNIPAM was used as the thermosensitive corona (56). The resulting pNIPAM-b-PBMA micelles were loaded with DOX and released $15 \%$ of the drug after $15 \mathrm{~h}$ at $30^{\circ} \mathrm{C}$, compared to $90 \%$ release in the same time period at $37^{\circ} \mathrm{C}$. Cytotoxicity experiments showed less than $5 \%$ cell death at $29^{\circ} \mathrm{C}$, but $65 \%$ cell death at $37^{\circ} \mathrm{C}$.

Presently, ultrasound is used to trigger drug release from drug delivery systems through mechanisms that include local temperature increase, cavitation which increases the permeability of cell membranes, and the production of highly reactive free radical species which can accelerate polymer degradation (57). Pitt and coworkers designed ultrasoundsensitive pluronic micelles containing doxorubicin $(58,59)$. Following stabilization of these pluronic micelles with PEG-phospholipid (PEG-DSPE), in vivo experiments showed that ultrasound was able to improve the antitumor efficacy of both free DOX and micelle incorporated DOX, with ultrasound delaying tumor growth significantly longer over micelles without ultrasound.

\section{Polymer Micelles with Imaging Sensitivity}

Medical imaging modalities such as magnetic resonance imaging (MRI), computed tomography (CT), single photon emission computed tomography (SPECT), positron emission tomography (PET), and ultrasonography play vital roles in cancer diagnosis and monitoring of therapy. Currently, the field of nanomedicine is converging with medical imaging to further increase contrast specificity between healthy and tumor tissues. 
Several micellar platforms have been established for use in MR imaging. Presently, gadolinium (Gd)-based contrast agents (e.g. Magnevist ${ }^{\circledR}$ ) are clinically used where image contrast is increased by shortening the $\mathrm{T}_{1}$ relaxation time of water protons (60). Incorporation of $\mathrm{Gd}$ complex on the surface of polymeric micelles can effectively increase the $\mathrm{T}_{1}$ relaxivity and sensitivity of detection $(61,62)$. Superparamagnetic iron oxide (SPIO) is a $\mathrm{T}_{2} / \mathrm{T}_{2}{ }^{*}$-based contrast agent that has higher sensitivity than Gd-based $\mathrm{T}_{1}$ agents (63). Our laboratory recently reported a formulation composed of polymeric micelles encapsulating a cluster of SPIO nanoparticles (64). Clustering of the hydrophobic SPIO nanoparticles inside of the micelle core led to a dramatic increase in $\mathrm{T}_{2}$ relaxivity. The micellar formulation showed MRI detection limit at nanomolar concentrations.

Polymeric micelles have also been explored for cancer imaging using CT and SPECT. Torchilin et al. developed iodine-containing PLL-PEG polymer micelles having an average diameter of $100 \mathrm{~nm}$ and iodine content of $45 \%$ (w/w) for use as CT contrast agents (65). Following subcutaneous injection in the hind leg of rabbits, they were able to identify popliteal lymph nodes after $2 \mathrm{~h}$ using CT. Opacification of the liver and spleen were observed after IV injection of the micelles into rabbits, with vessels in the liver being demarcated within half an hour of injection.

\section{Multifunctional Polymeric Micelles}

The unique architecture of polymeric micelles allows for the incorporation of multiple functional components within a single micelle. By combining tumor targeting, stimulated release of therapeutics, and the delivery of imaging agents, multiple interventions against a tumor can be integrated into one platform. Such a 'theranostic' entity, has been defined as a nanomedicine platform that can diagnose, deliver targeted treatment in a controlled manner, and monitor response to cancer therapy (66).

Kataoka and coworkers explored the use of multifunctional micelles by conjugating a targeting ligand, folate, to $\mathrm{pH}$-sensitive doxorubicin-releasing polymer micelles composed of PEG-p (Asp-Hyd-ADR) (67). The multifunctional platform proved more effective at treating KB cells (nearly 10 -fold after a $24 \mathrm{~h}$ exposure) than the untargeted formulation. Similarly, when folate was introduced to their pH-sensitive PEG-pHis DOX-containing micelles, Bae and coworkers observed three times greater accumulation in MCF-7 tumors in vivo and greater cytotoxicity compared to folate-free micelles (68). An alternate micellar platform developed by Kennedy and coworkers combined delivery of doxorubicin and imaging of tumors via ultrasound (69). In this strategy, consisting of DOX-containing PEG-PLLA micelles and nanodroplets of perfluoropentane (PFP) stabilized by an outer layer of block copolymer, ultrasound triggered drug release from micelles through inertial cavitation and facilitated entry into tumor cells by altering cell permeability. Upon i.v. administration of the formulation, MDA-MB 231 tumors not treated with ultrasound showed a pattern of growth similar to control tumors. Tumors undergoing administration of the micelles and treated with ultrasound (30 second treatment at $3 \mathrm{MHz}$ ) showed significant regression. Additionally, within 4 hours after injection, ultrasonography revealed strong echoes within the center of the tumor, further demonstrating in vivo coalescing of nanobubbles.

Recently, a multifunctional micellar platform that incorporates a targeting ligand, $\mathrm{pH}$ stimulated release of DOX, and an MRI-visible agent was established in our laboratory (70). In this design, DOX and a cluster of SPIO nanoparticles ( $8 \mathrm{~nm}$ in diameter) were loaded into the cores of PEG-PLA micelles, while a cRGD ligand on the micelle surface (cRGD-DOXSPIO micelles) was used for targeting as shown in Figure 3A. The resulting targeted, multifunctional micelles, measuring $45 \pm 8 \mathrm{~nm}$ in diameter showed increased uptake in vitro in $\alpha_{v} \beta_{3}$-overexpressing SLK endothelial cells. 
In in vivo studies, the multifunctional micelles showed effective tumor targeting, imaging sensitivity, and antitumor efficacy. Figure 3B shows a pre-contrast image obtained using a $\mathrm{T}_{2}$-weighted $\left(\mathrm{T}_{2}-\mathrm{w}\right)$ scan on a $4.7 \mathrm{~T}$ MRI scanner. The coronal image depicts the presence of an orthotopic H1299 non-small cell lung tumor xenograft. Following an i.v. injection of a solution of DOXO-SPIO micelles with $16 \%$ cRGD surface density $(6.3 \mathrm{mg} \mathrm{Fe} / \mathrm{kg}), \mathrm{a} \mathrm{T}_{2}$-w image taken 15 hours after injection demonstrates significant darkening of the tumor (Fig. 3C), showing micelle accumulation in tumors. The antitumor efficacy of the micelle platform in the orthotopic H1299 tumor model was monitored by bioluminescent imaging (BLI) of tumors transfected with the firefly luciferase gene. Figure 3D represents a BLI image of a representative mouse at day 0 , immediately before the micelle injection, showing a relative luminescence unit (RLU) value of $1.2 \times 10^{6}$. At days 1 and 3, a solution of cRGD-encoded DOXO-SPIO micelles (4 mg DOX/kg) was injected intravenously. At day 7, BLI was performed and the RLU value was found to be $5.6 \times 10^{5}$, indicating an approximate $50 \%$ reduction in tumor size. In a separate antitumor efficacy study, a subcutaneous A549 lung tumor xenograft was utilized (Figure 3F). As in the case of the orthotopic study, cRGD-encoded DOX-SPIO micelles exhibited regression of tumor size (approximately 50\% size reduction after 10 days). On the contrary, tumor sizes from mice treated with cRGD-free DOX-SPIO micelles did not change dramatically, with the PBS control group showing a steady tumor growth, the size almost doubling after 10 days.

\section{Conclusions and Future Outlook}

Polymeric micelles are emerging as a multifunctional nanotherapeutic platform for cancer imaging and therapeutic applications. Herein, we summarized recent advances in micellar nanomedicine capable of targeting tumors through the addition of targeting ligands while delivering anti-neoplastic drugs with responsive release to achieve maximal antitumor efficacy. Furthermore, micelles incorporating imaging functions are established for diagnostic and monitoring purposes to assess therapeutic efficacy. The incorporation of multiple functionalities in polymeric micelles is a necessary step towards overcoming the biological complexity and therapeutic challenges during cancer chemotherapy. Solid tumors are known to have a highly heterogeneous population of different cell types. Molecular and phenotypic heterogeneity becomes a significant challenge since not all cells within a given tumor will respond to a single therapeutic agent. Additionally, as more specific agents are developed to target tumors, selection of tumor cells that can bypass the targeted pathway will lead to adaptive resistance and become a cause of treatment failure. As such, micellar nanomedicine that can deliver multiple agents to target several key cancerous pathways will be the next step in their evolution. To further overcome the challenge of tumor heterogeneity, micelles with imaging functions to characterize the phenotypic expressions within solid tumors will be valuable in guiding the selection of tumor-specific therapy. In addition, such imaging functions allow the monitoring of treatment efficacy and enable the subsequent modification of therapy to address adaptive resistance. Such theranostic micellar nanomedicine holds considerable promise to achieve personalized therapy of cancer to maximize the specificity and efficacy of chemotherapy.

\section{Acknowledgments}

This work is supported by an NIH RO1 grant (CA 122994) to JG. EB is supported by a DOD predoctoral grant W81XWH-05-1-0258. This is report 0035 from the Cell Stress and Cancer Nanomedicine program in the Simmons Comprehensive Cancer Center at the University of Texas Southwestern Medical Center at Dallas.

\section{References}

1. Duncan R. The dawning era of polymer therapeutics. Nat Rev Drug Discov 2003;2:347-360. [PubMed: 12750738] 
2. Ferrari M. Cancer nanotechnology: opportunities and challenges. Nat Rev Cancer 2005;5:161-171. [PubMed: 15738981]

3. Peer D, Karp JM, Hong S, Farokhzad OC, Margalit R, Langer R. Nanocarriers as an emerging platform for cancer therapy. Nat Nanotechnol 2007;2:751-760. [PubMed: 18654426]

4. Croy SR, Kwon GS. Polymeric micelles for drug delivery. Curr Pharm Des 2006;12:4669-4684. [PubMed: 17168771]

5. Nishiyama N, Kataoka K. Current state, achievements, and future prospects of polymeric micelles as nanocarriers for drug and gene delivery. Pharmacol Ther 2006;112:630-648. [PubMed: 16815554]

6. Sutton D, Nasongkla N, Blanco E, Gao J. Functionalized micellar systems for cancer targeted drug delivery. Pharm Res 2007;24:1029-1046. [PubMed: 17385025]

7. Torchilin VP. Micellar nanocarriers: pharmaceutical perspectives. Pharm Res 2007;24:1-16. [PubMed: 17109211]

8. Gros L, Ringsdorf H, Schupp H. Polymeric Anti-Tumor Agents on a Molecular and on a CellularLevel. Angew Chem Int Edit 1981;20:305-325.

9. Jones M, Leroux J. Polymeric micelles - a new generation of colloidal drug carriers. Eur J Pharm Biopharm 1999;48:101-111. [PubMed: 10469928]

10. Torchilin VP. Structure and design of polymeric surfactant-based drug delivery systems. J Control Release 2001;73:137-172. [PubMed: 11516494]

11. Torchilin VP, Lukyanov AN, Gao Z, Papahadjopoulos-Sternberg B. Immunomicelles: targeted pharmaceutical carriers for poorly soluble drugs. Proc Natl Acad Sci U S A 2003;100:6039-6044. [PubMed: 12716967]

12. Soga O, van Nostrum CF, Fens M, Rijcken CJ, Schiffelers RM, Storm G, Hennink WE. Thermosensitive and biodegradable polymeric micelles for paclitaxel delivery. J Control Release 2005;103:341-353. [PubMed: 15763618]

13. Moghimi SM, Hunter AC. Recognition by macrophages and liver cells of opsonized phospholipid vesicles and phospholipid headgroups. Pharm Res 2001;18:1-8. [PubMed: 11336343]

14. Torchilin VP. PEG-based micelles as carriers of contrast agents for different imaging modalities. Adv Drug Deliv Rev 2002;54:235-252. [PubMed: 11897148]

15. Liu J, Zeng F, Allen C. In vivo fate of unimers and micelles of a poly(ethylene glycol)-block-poly (caprolactone) copolymer in mice following intravenous administration. Eur J Pharm Biopharm 2007;65:309-319. [PubMed: 17257817]

16. Hashizume H, Baluk P, Morikawa S, McLean JW, Thurston G, Roberge S, Jain RK, McDonald DM. Openings between defective endothelial cells explain tumor vessel leakiness. Am J Pathol 2000;156:1363-1380. [PubMed: 10751361]

17. Maeda $H$. The enhanced permeability and retention (EPR) effect in tumor vasculature: the key role of tumor-selective macromolecular drug targeting. Adv Enzyme Regul 2001;41:189-207. [PubMed: 11384745]

18. Li C, Wallace S. Polymer-drug conjugates: Recent development in clinical oncology. Adv Drug Deliv Rev. 2008

19. Lee CC, MacKay JA, Frechet JM, Szoka FC. Designing dendrimers for biological applications. Nat Biotechnol 2005;23:1517-1526. [PubMed: 16333296]

20. Torchilin VP. Recent advances with liposomes as pharmaceutical carriers. Nat Rev Drug Discov 2005;4:145-160. [PubMed: 15688077]

21. Maeda H, Ueda M, Morinaga T, Matsumoto T. Conjugation of poly(styrene-co-maleic acid) derivatives to the antitumor protein neocarzinostatin: pronounced improvements in pharmacological properties. J Med Chem 1985;28:455-461. [PubMed: 3156994]

22. Greish K, Fang J, Inutsuka T, Nagamitsu A, Maeda H. Macromolecular therapeutics: advantages and prospects with special emphasis on solid tumour targeting. Clin Pharmacokinet 2003;42:1089-1105. [PubMed: 14531722]

23. Gabizon AA. Pegylated liposomal doxorubicin: metamorphosis of an old drug into a new form of chemotherapy. Cancer Invest 2001;19:424-436. [PubMed: 11405181] 
24. Kukowska-Latallo JF, Candido KA, Cao Z, Nigavekar SS, Majoros IJ, Thomas TP, Balogh LP, Khan MK, Baker JR Jr. Nanoparticle targeting of anticancer drug improves therapeutic response in animal model of human epithelial cancer. Cancer Res 2005;65:5317-5324. [PubMed: 15958579]

25. Nakanishi T, Fukushima S, Okamoto K, Suzuki M, Matsumura Y, Yokoyama M, Okano T, Sakurai Y, Kataoka K. Development of the polymer micelle carrier system for doxorubicin. J Control Release 2001;74:295-302. [PubMed: 11489509]

26. Matsumura Y, Hamaguchi T, Ura T, Muro K, Yamada Y, Shimada Y, Shirao K, Okusaka T, Ueno H, Ikeda M, Watanabe N. Phase I clinical trial and pharmacokinetic evaluation of NK911, a micelleencapsulated doxorubicin. Br J Cancer 2004;91:1775-1781. [PubMed: 15477860]

27. Gillies ER, Frechet JM. Dendrimers and dendritic polymers in drug delivery. Drug Discov Today 2005;10:35-43. [PubMed: 15676297]

28. Gopin A, Ebner S, Attali B, Shabat D. Enzymatic activation of second-generation dendritic prodrugs: Conjugation of self-immolative dendrimers with poly(ethylene glycol) via click chemistry.

Bioconjug Chem 2006;17:1432-1440. [PubMed: 17105221]

29. Greenwald RB, Choe YH, McGuire J, Conover CD. Effective drug delivery by PEGylated drug conjugates. Adv Drug Deliv Rev 2003;55:217-250. [PubMed: 12564978]

30. Liu J, Lee H, Huesca M, Young A, Allen C. Liposome formulation of a novel hydrophobic arylimidazole compound for anti-cancer therapy. Cancer Chemother Pharmacol 2006;58:306-318. [PubMed: 16333677]

31. Kamaly N, Kalber T, Ahmad A, Oliver MH, So PW, Herlihy AH, Bell JD, Jorgensen MR, Miller AD. Bimodal paramagnetic and fluorescent liposomes for cellular and tumor magnetic resonance imaging. Bioconjug Chem 2008;19:118-129. [PubMed: 17985841]

32. Zalipsky S, Saad M, Kiwan R, Ber E, Yu N, Minko T. Antitumor activity of new liposomal prodrug of mitomycin $\mathrm{C}$ in multidrug resistant solid tumor: insights of the mechanism of action. J Drug Target 2007;15:518-530. [PubMed: 17671898]

33. Yuan F, Leunig M, Huang SK, Berk DA, Papahadjopoulos D, Jain RK. Microvascular permeability and interstitial penetration of sterically stabilized (stealth) liposomes in a human tumor xenograft. Cancer Res 1994;54:3352-3356. [PubMed: 8012948]

34. McKee TD, Grandi P, Mok W, Alexandrakis G, Insin N, Zimmer JP, Bawendi MG, Boucher Y, Breakefield XO, Jain RK. Degradation of fibrillar collagen in a human melanoma xenograft improves the efficacy of an oncolytic herpes simplex virus vector. Cancer Res 2006;66:2509-2513. [PubMed: 16510565]

35. Chen Q, Tong S, Dewhirst MW, Yuan F. Targeting tumor microvessels using doxorubicin encapsulated in a novel thermosensitive liposome. Mol Cancer Ther 2004;3:1311-1317. [PubMed: 15486198]

36. Li W, Huang Z, MacKay JA, Grube S, Szoka FC Jr. Low-pH-sensitive poly(ethylene glycol) (PEG)stabilized plasmid nanolipoparticles: effects of PEG chain length, lipid composition and assembly conditions on gene delivery. J Gene Med 2005;7:67-79. [PubMed: 15515149]

37. Bae Y, Nishiyama N, Fukushima S, Koyama H, Yasuhiro M, Kataoka K. Preparation and biological characterization of polymeric micelle drug carriers with intracellular $\mathrm{pH}$-triggered drug release property: tumor permeability, controlled subcellular drug distribution, and enhanced in vivo antitumor efficacy. Bioconjug Chem 2005;16:122-130. [PubMed: 15656583]

38. Yoo HS, Park TG. Folate receptor targeted biodegradable polymeric doxorubicin micelles. J Control Release 2004;96:273-283. [PubMed: 15081218]

39. Shuai X, Ai H, Nasongkla N, Kim S, Gao J. Micellar carriers based on block copolymers of poly (epsilon-caprolactone) and poly(ethylene glycol) for doxorubicin delivery. J Control Release 2004;98:415-426. [PubMed: 15312997]

40. Allen C, Maysinger D, Eisenberg A. Nano-engineering block copolymer aggregates for drug delivery. Colloid Surface B 1999;16:3-27.

41. Gaucher G, Dufresne MH, Sant VP, Kang N, Maysinger D, Leroux JC. Block copolymer micelles: preparation, characterization and application in drug delivery. J Control Release 2005;109:169-188. [PubMed: 16289422] 
42. Kim SY, Shin IG, Lee YM. Preparation and characterization of biodegradable nanospheres composed of methoxy poly(ethylene glycol) and DL-lactide block copolymer as novel drug carriers. J Control Release 1998;56:197-208. [PubMed: 9801443]

43. Liu J, Xiao Y, Allen C. Polymer-drug compatibility: a guide to the development of delivery systems for the anticancer agent, ellipticine. J Pharm Sci 2004;93:132-143. [PubMed: 14648643]

44. Bontha S, Kabanov AV, Bronich TK. Polymer micelles with cross-linked ionic cores for delivery of anticancer drugs. J Control Release 2006;114:163-174. [PubMed: 16914223]

45. Xu P, Tang H, Li S, Ren J, Van Kirk E, Murdoch WJ, Radosz M, Shen Y. Enhanced stability of coresurface cross-linked micelles fabricated from amphiphilic brush copolymers. Biomacromolecules 2004;5:1736-1744. [PubMed: 15360282]

46. Sethuraman VA, Bae YH. TAT peptide-based micelle system for potential active targeting of anticancer agents to acidic solid tumors. J Control Release 2007;118:216-224. [PubMed: 17239466]

47. Ross JF, Chaudhuri PK, Ratnam M. Differential regulation of folate receptor isoforms in normal and malignant tissues in vivo and in established cell lines. Physiologic and clinical implications. Cancer 1994;73:2432-2443.

48. Silletti S, Kessler T, Goldberg J, Boger DL, Cheresh DA. Disruption of matrix metalloproteinase 2 binding to integrin alpha vbeta 3 by an organic molecule inhibits angiogenesis and tumor growth in vivo. Proc Natl Acad Sci U S A 2001;98:119-124. [PubMed: 11134507]

49. Wands JR, Blum HE. Primary hepatocellular carcinoma. N Engl J Med 1991;325:729-731. [PubMed: 1651454]

50. Jeong YI, Seo SJ, Park IK, Lee HC, Kang IC, Akaike T, Cho CS. Cellular recognition of paclitaxelloaded polymeric nanoparticles composed of poly(gamma-benzyl L-glutamate) and poly(ethylene glycol) diblock copolymer endcapped with galactose moiety. Int J Pharm 2005;296:151-161. [PubMed: 15885467]

51. Farokhzad OC, Cheng J, Teply BA, Sherifi I, Jon S, Kantoff PW, Richie JP, Langer R. Targeted nanoparticle-aptamer bioconjugates for cancer chemotherapy in vivo. Proc Natl Acad Sci U S A 2006;103:6315-6320. [PubMed: 16606824]

52. Potineni A, Lynn DM, Langer R, Amiji MM. Poly(ethylene oxide)-modified poly(beta-amino ester) nanoparticles as a $\mathrm{pH}$-sensitive biodegradable system for paclitaxel delivery. J Control Release 2003;86:223-234. [PubMed: 12526819]

53. Stapert HR, Nishiyama N, Jiang DL, Aida T, Kataoka K. Polyion complex micelles encapsulating light-harvesting ionic dendrimer zinc porphyrins. Langmuir 2000;16:8182-8188.

54. Tang Y, Liu SY, Armes SP, Billingham NC. Solubilization and controlled release of a hydrophobic drug using novel micelle-forming ABC triblock copolymers. Biomacromolecules 2003;4:16361645. [PubMed: 14606890]

55. Liu SQ, Tong YW, Yang YY. Thermally sensitive micelles self-assembled from poly $(\mathrm{N}-$ isopropylacrylamide-co-N,N-dimethylacrylamide)-b-poly(D,L-lactide-c o-glycolide) for controlled delivery of paclitaxel. Mol Biosyst 2005;1:158-165. [PubMed: 16880979]

56. Chung JE, Yokoyama M, Yamato M, Aoyagi T, Sakurai Y, Okano T. Thermo-responsive drug delivery from polymeric micelles constructed using block copolymers of poly(Nisopropylacrylamide) and poly(butylmethacrylate). J Control Release 1999;62:115-127. [PubMed: 10518643]

57. Mitragotri S. Healing sound: the use of ultrasound in drug delivery and other therapeutic applications. Nat Rev Drug Discov 2005;4:255-260. [PubMed: 15738980]

58. Gao ZG, Fain HD, Rapoport N. Controlled and targeted tumor chemotherapy by micellarencapsulated drug and ultrasound. J Control Release 2005;102:203-222. [PubMed: 15653146]

59. Pruitt JD, Pitt WG. Sequestration and ultrasound-induced release of doxorubicin from stabilized Pluronic P105 micelles. Drug Deliv 2002;9:253-258. [PubMed: 12511204]

60. Sato H, Enmi J, Teramoto N, Hayashi T, Yamamoto A, Tsuji T, Naito H, Iida H. Comparison of GdDTPA-induced signal enhancements in rat brain C6 glioma among different pulse sequences in 3Tesla magnetic resonance imaging. Acta Radiol 2008;49:172-179. [PubMed: 18300142]

61. Nakamura E, Makino K, Okano T, Yamamoto T, Yokoyama M. A polymeric micelle MRI contrast agent with changeable relaxivity. J Control Release 2006;114:325-333. [PubMed: 16891027] 
62. Zhang G, Zhang R, Wen X, Li L, Li C. Micelles based on biodegradable poly(L-glutamic acid)-bpolylactide with paramagnetic Gd ions chelated to the shell layer as a potential nanoscale MRI-visible delivery system. Biomacromolecules 2008;9:36-42. [PubMed: 18047289]

63. Wang YX, Hussain SM, Krestin GP. Superparamagnetic iron oxide contrast agents: physicochemical characteristics and applications in MR imaging. Eur Radiol 2001;11:2319-2331. [PubMed: 11702180]

64. Ai H, Flask C, Weinberg B, Shuai X, Pagel MD, Farrell D, Duerk J, Gao J. Magnetite-loaded polymeric micelles as ultrasensitive magnetic-resonance probes. Adv Mater 2005;17:1949-1952.

65. Trubetskoy VS, Gazelle GS, Wolf GL, Torchilin VP. Block-copolymer of polyethylene glycol and polylysine as a carrier of organic iodine: design of long-circulating particulate contrast medium for X-ray computed tomography. J Drug Target 1997;4:381-388. [PubMed: 9239578]

66. Sumer B, Gao J. Theranostic nanomedicine for cancer. Nanomed 2008;3:137-140.

67. Bae Y, Jang WD, Nishiyama N, Fukushima S, Kataoka K. Multifunctional polymeric micelles with folate-mediated cancer cell targeting and $\mathrm{pH}$-triggered drug releasing properties for active intracellular drug delivery. Mol Biosyst 2005;1:242-250. [PubMed: 16880988]

68. Lee ES, Na K, Bae YH. Doxorubicin loaded pH-sensitive polymeric micelles for reversal of resistant MCF-7 tumor. J Control Release 2005;103:405-418. [PubMed: 15763623]

69. Rapoport N, Gao Z, Kennedy A. Multifunctional nanoparticles for combining ultrasonic tumor imaging and targeted chemotherapy. J Natl Cancer Inst 2007;99:1095-1106. [PubMed: 17623798]

70. Nasongkla N, Bey E, Ren J, Ai H, Khemtong C, Guthi JS, Chin SF, Sherry AD, Boothman DA, Gao J. Multifunctional polymeric micelles as cancer-targeted, MRI-ultrasensitive drug delivery systems. Nano Lett 2006;6:2427-2430. [PubMed: 17090068] 


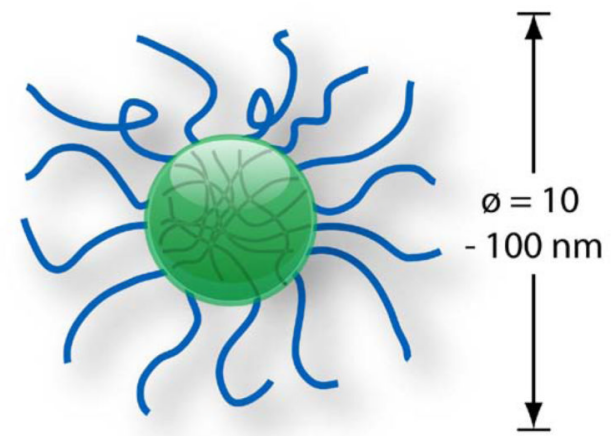

amphiphilic polymer:

hydrophilic block

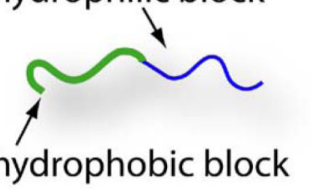

Figure 1.

Schematic of the core-shell architecture of a polymer micelle and its dimensions. 

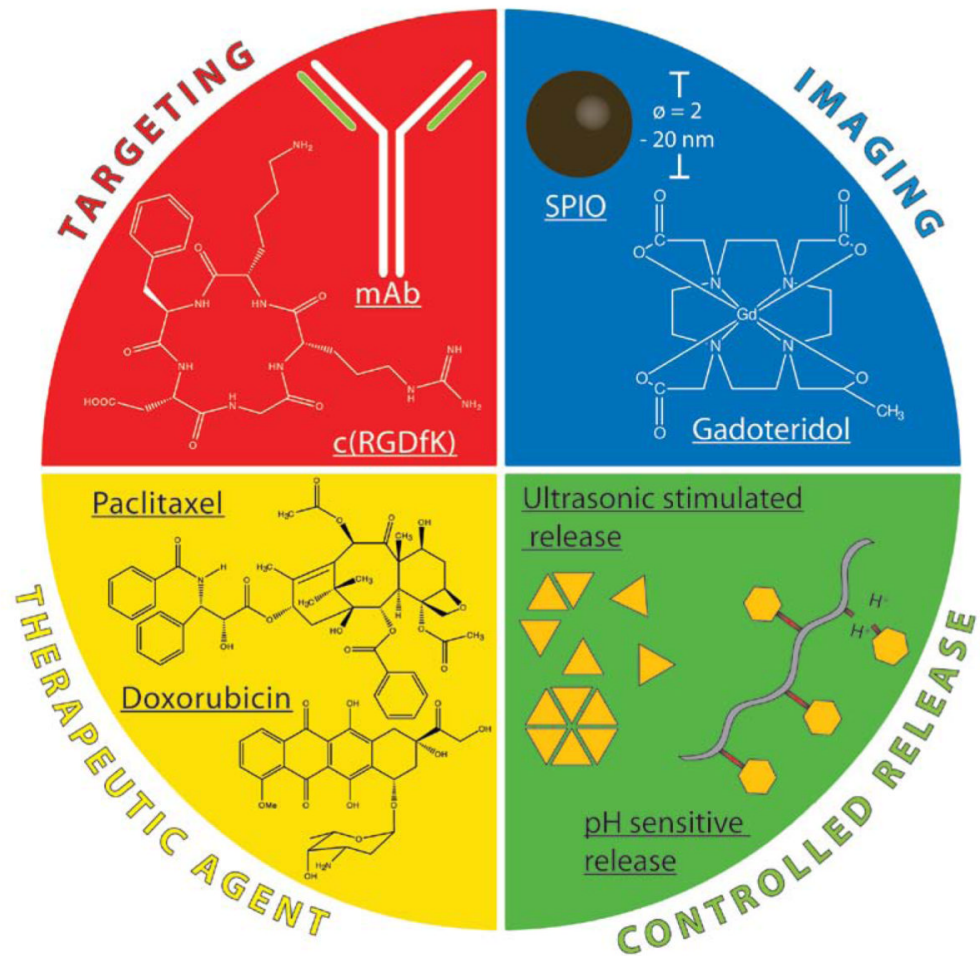

Figure 2.

Multifunctional design of a micelle nanomedicine platform with cancer targeting, imaging, and controlled release properties. 
A
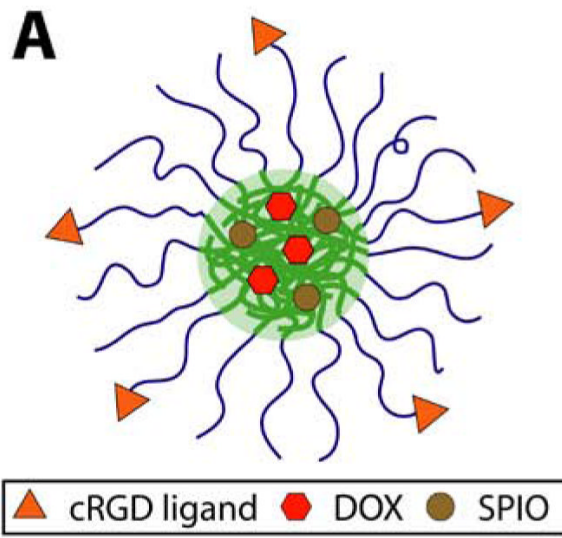

\section{D}

Figure 3.
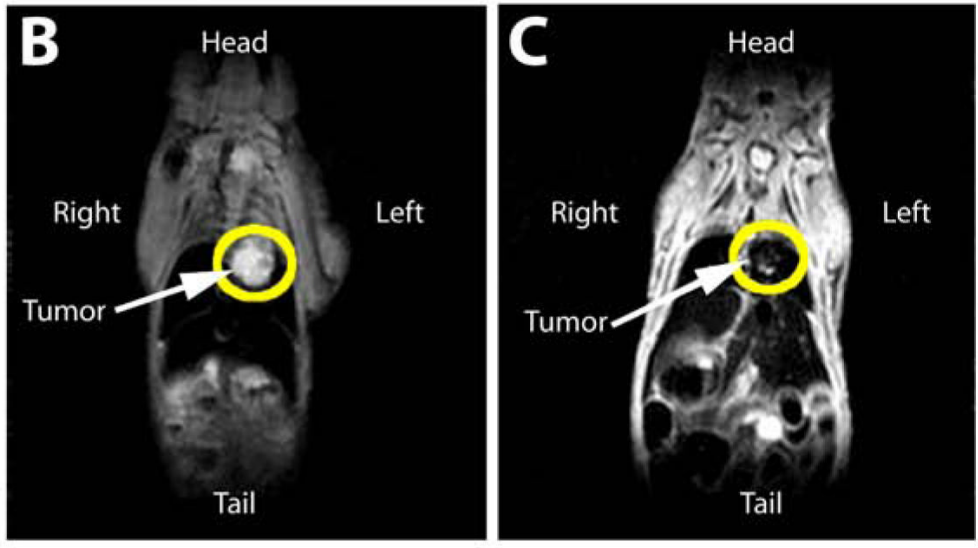

$\mathbf{F}$

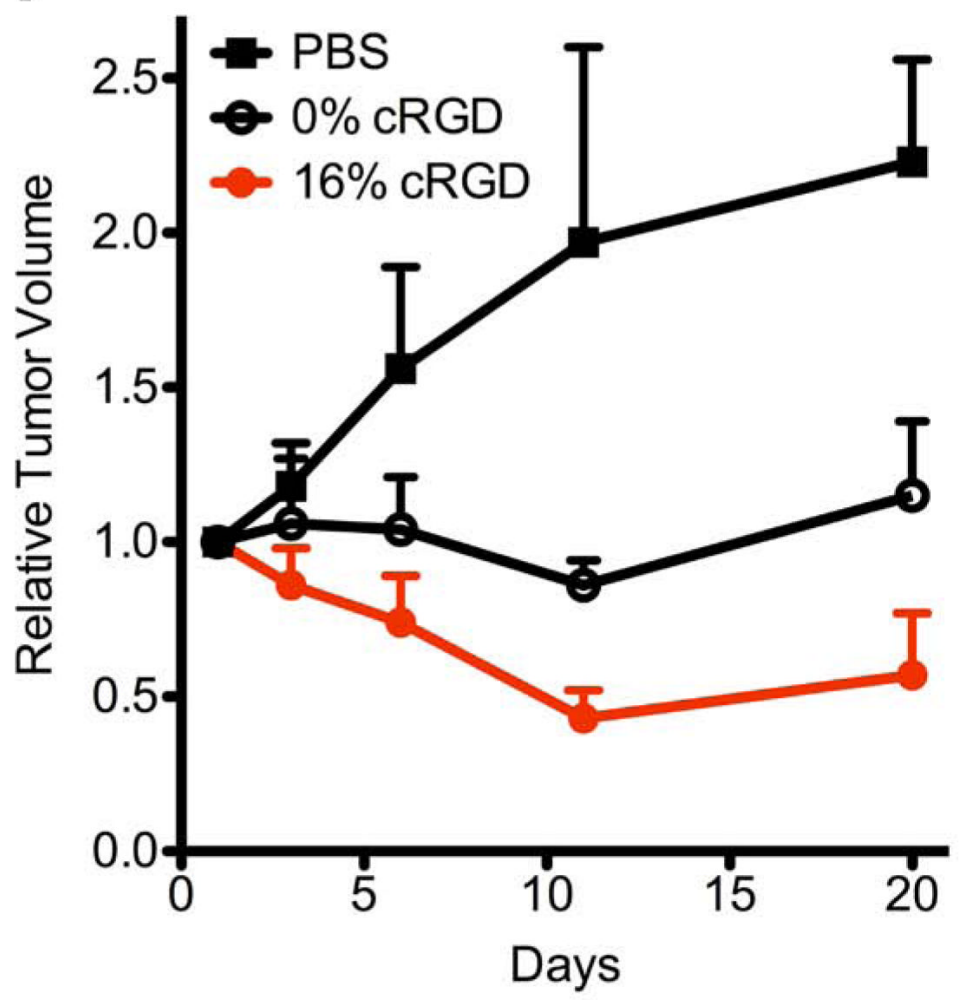

(A) Schematic of a multifunctional polymeric micelle. (B-C) Pre- and post-contrast coronal images of tumor-bearing mice by $\mathrm{T}_{2}$-weighted imaging ( $\mathrm{TR} / \mathrm{TE}=4 \mathrm{~s} / 40 \mathrm{~ms}$ ), respectively. (DE) Bioluminescence imaging of mouse bearing luciferase-transfected H1299 xenograft at day 0 and 7 , respectively. DOX-loaded, 16\% cRGD-micelles were injected via tail vein at day 0 and 3 (4 mg/kg DOX dose each time). (F) Antitumor efficacy data for PBS control, DOXOSPIO micelles with 0 and $16 \%$ surface density of cRGD in subcutaneous A549 tumor xenografts in nude mice. Each data point is averaged from 3 animals. 
Table 1

Summary of different nanotherapeutic technologies proposed for cancer therapy

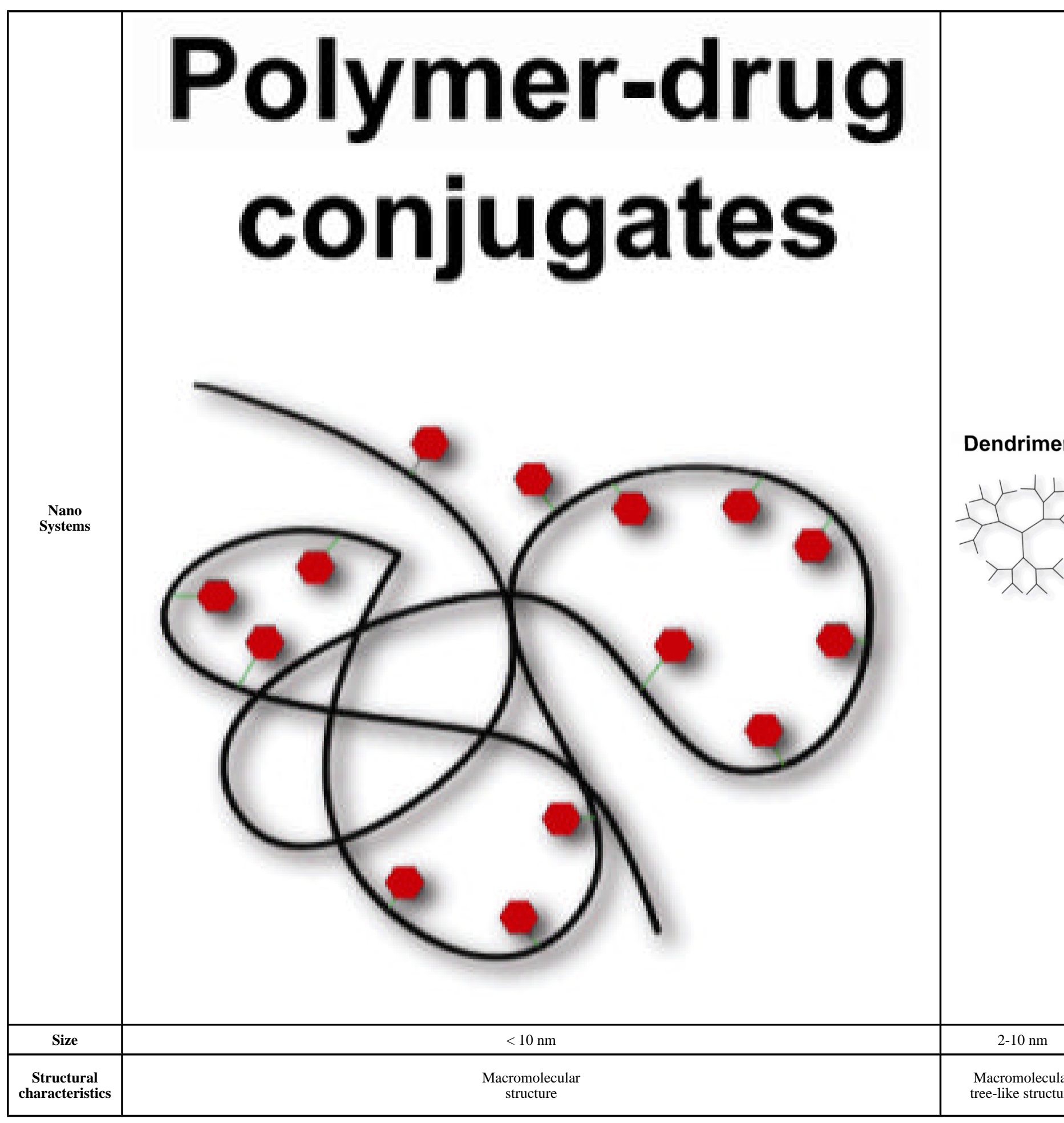

Exp Biol Med (Maywood). Author manuscript; available in PMC 2010 May 6. 


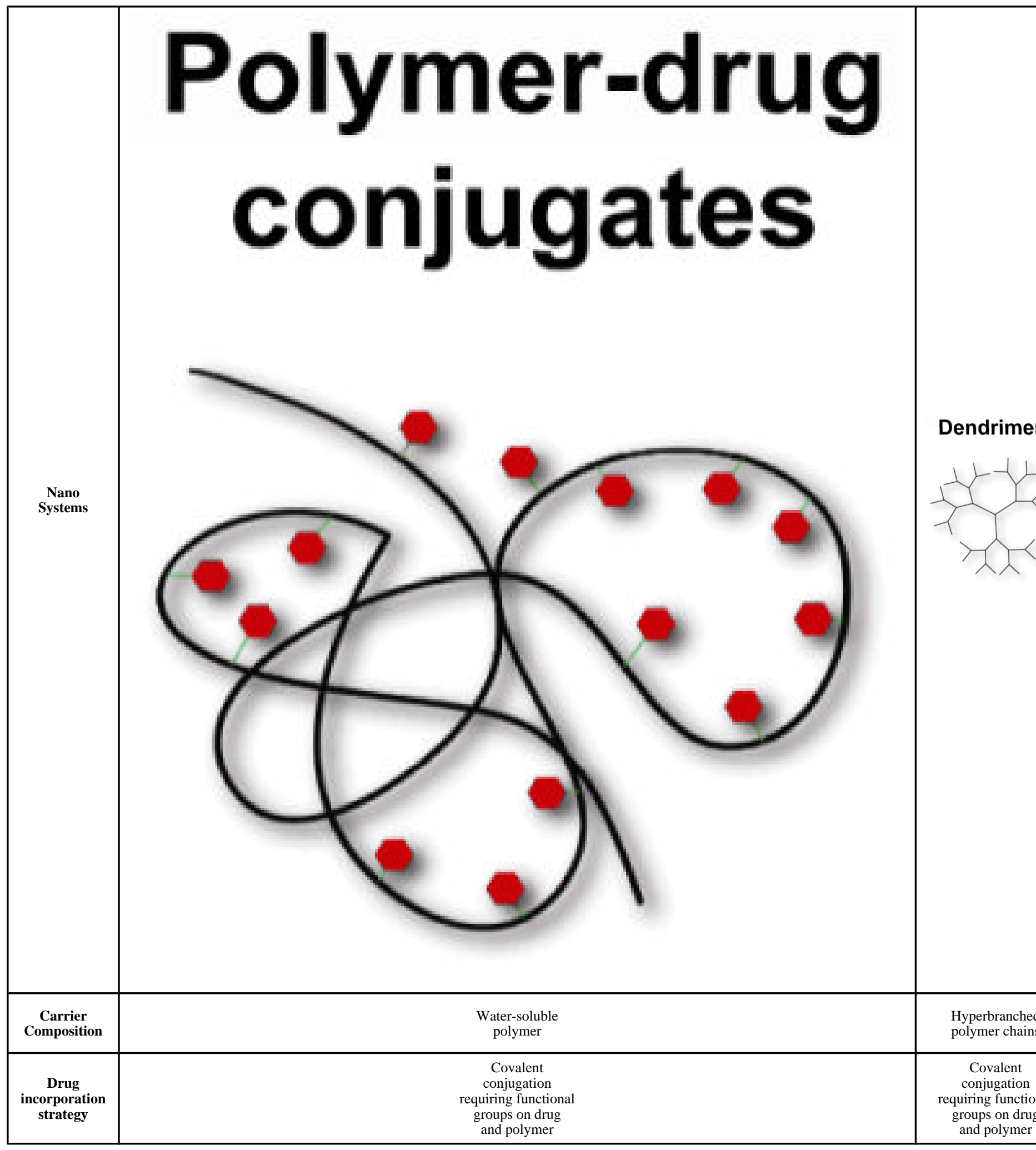

Exp Biol Med (Maywood). Author manuscript; available in PMC 2010 May 6. 


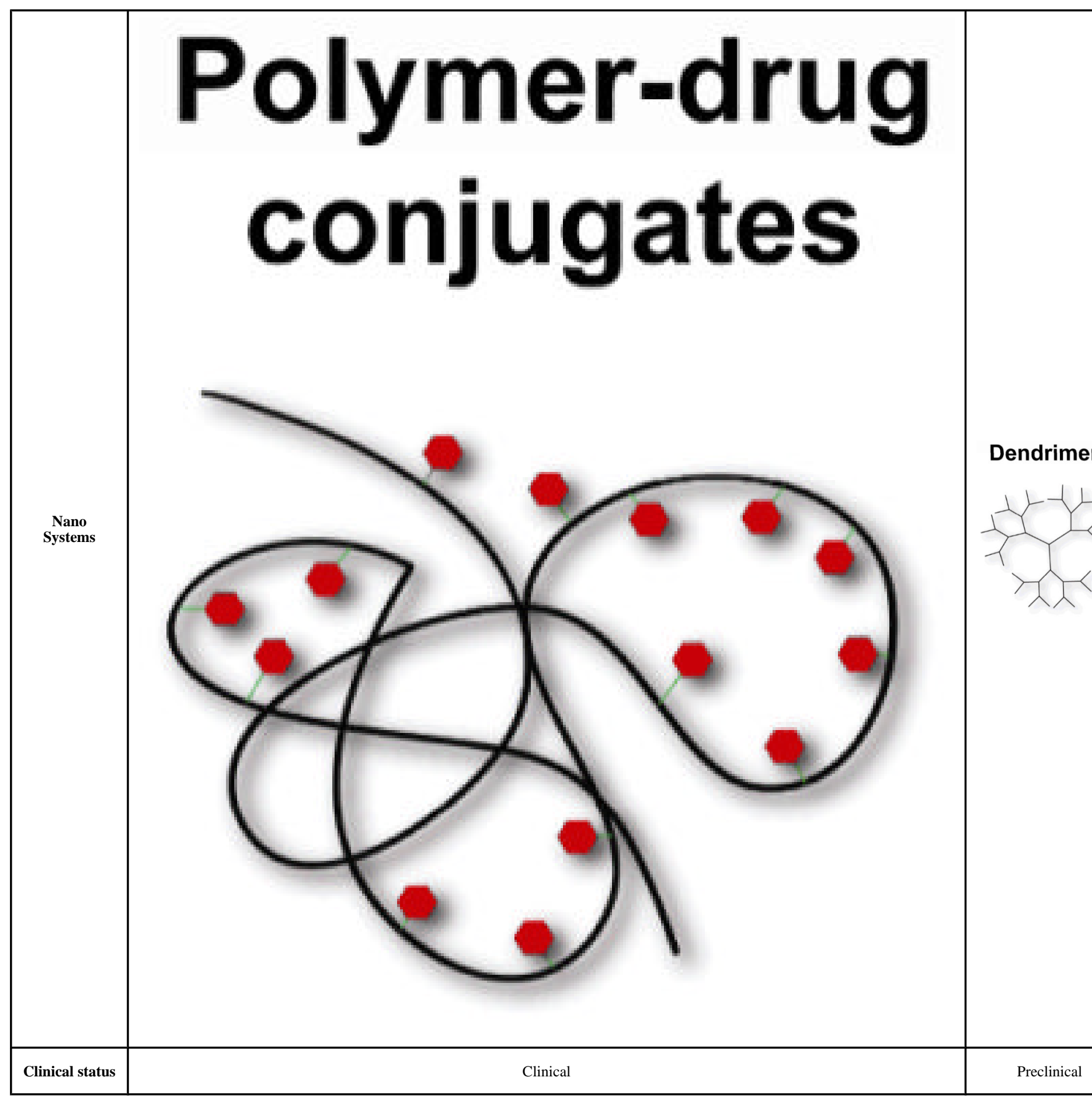

Exp Biol Med (Maywood). Author manuscript; available in PMC 2010 May 6. 